\title{
Cordyceps Mushroom: A Potent Anticancer Nutraceutical
}

\author{
Md. Asaduzzaman Khan ${ }^{1}$, Mousumi Tania ${ }^{1}$, Dian-zheng Zhang ${ }^{1,2}$ and Han-chun Chen ${ }^{1, *}$ \\ ${ }^{I}$ Department of Biochemistry, School of Biological Science and Technology, Central South University, Changsha, \\ Hunan 410013, P R China; ${ }^{2}$ Department of Biochemistry/Molecular Biology and Center for Chronic Disorders of \\ Aging, Philadelphia College of Osteopathic Medicine, Philadelphia, PA 19131, USA
}

\begin{abstract}
The Cordyceps mushrooms have a long history as medicinal fungi. In Traditional Chinese Medicine, Cordyceps have been used to treat several conditions including cancers for thousand of years. Extracts from both mycelium and fruiting bodies of $C$. sinensis, $C$. militaris and other Cordyceps species showed significant anticancer activities by various mechanisms such as, modulating immune system and inducing cell apoptosis. Some polysaccharide components and cordycepin (3'-deoxyadenosine) have been isolated from $C$. sinensis and $C$. militaris, which acted as potent anticancer components. This review article aims to further elucidate the importance of Cordyceps mushrooms by summarizing the findings of some of the important research works concerning possible mechanism of anticancer activity of this mushroom.
\end{abstract}

Keywords: C. sinensis, C. militaris, Cordycepin, Apoptosis, Immunomodulating, Anticancer activity.

\section{INTRODUCTION}

There is a common saying, 'Medicines and foods have a common origin'. Mushrooms are a manifestation of this idea in constituting both a nutritionally functional food and a source of physiologically beneficial medicine. Many centuries ago, medicinal properties of mushrooms have been recognized in China, Korea and Japan. Although from ancient times, mushrooms have been treated as a special kind of nutraceutical, they have received a remarkable interest in recent decades. Major medicinal properties attributed to mushrooms include anticancer activity, antibiotic activity (directed against bacteria, fungi and protozoa), antiviral activity, immune response-stimulating effects, anti-hypertensive and blood lipid lowering effects [1,2]. Some mushrooms have gained special consideration due to their various medicinal values in addition to nutritional importance. For example, Lentinus edodes were reported to possess anti-tumor, antihypertensive, hypocholesterolemic and antibacterial activities [3-6]. Ganoderma lucidum has been proved to have anti microbial and anti-HIV effects [7, 8]. The hepatic and renal protective effects of $G$. lucidum in mice were also evaluated [9]. The beta-glucan polysaccharide of this mushroom has potential application in immune surveillance and chemoprevention of cancer [2]. Mushrooms of Pleurotus species (P. ostreatus, P. sajor-caju, $P$. florida) were reported to have hypocholesterolemic activity in experimental rats $[10,11]$.

Cordyceps mushroom is a genus of ascomycete fungi that includes about 400 described species. All Cordyceps species are endoparasitoids, mainly on insects and other arthropods [12] These mushrooms have a long history as rare and exotic

\footnotetext{
*Address correspondence to this author at the Department of Biochemistry, School of Biological Science and Technology, (172 Tongzipo Rd., Xiangya School of Medicine), Central South University, Changsha, Hunan 410013, P R China; Tel: 86-731-82650411; Fax: 86-731-82650230;

E-mail: chenhanchun@mail.csu.edu.cn
}

medicinal fungi. They have been a highly regarded cornerstone of traditional Chinese medicine for centuries; that apparently have a number of far reaching medicinal effects [13, 14]. Cordyceps mushrooms have been used to treat conditions including respiration and pulmonary diseases; renal, liver, and cardiovascular diseases; hyposexuality and hyperlipidemia. It is also used in the treatment of immune disorders and as an adjunct to modern cancer therapies (chemotherapy, radiation treatment and surgery) [12]. C. sinensis and $C$. militaris are the most common in use among the Cordyceps genus.

\section{ANTICANCER ACTIVITY OF CORDYCEPS SINENSIS}

C. sinensis is a fungus that has been used for over 2000 years in China as a treatment for a variety of conditions including cancer. Many available evidences suggest that the efficacy of $C$. sinensis as an anti-neoplastic therapeutic agent is due to its role as an activator of immune responses. Extracts from both mycelium and fruiting bodies from $C$. sinensis influence the immune system in various ways. In a study, water-extract from dried $C$. sinensis increased the median survival time of the allogeneic mice inoculated with Ehrlich ascites carcinoma cells (EAC) to $316 \%$ and syngeneic mice inoculated with Meth A fibrosarcoma (Meth A) to $312 \%$ of the control with no cytotoxic activity on either EAC or Meth A in vitro [15]. The water extract of this mushroom has been also proved beneficial in the prevention of tumor metastasis in mice as an adjuvant agent in cancer chemotherapy [16]. The natural killer cells (NK) activities of mouse were both in vivo and in vitro significantly augmented by intraperitoneal injection of the ethanol extract of $C$. sinensis [17]. The ethanol-extract also significantly decreased tumor weights and volumes in mice inoculated with Sarcoma-180 tumor cells. The extracts were demonstrated to exhibit phagocytosis enhancing activity as measured by carbon clearance in mice and caused a significant increase in an acid phosphatase 
activity, representing lysosomal enzymes, suggesting that the anti-tumor activity of these fungi might be related to an immuno-stimulating function [18]. The ethyl acetate extract of C. sinensis mycelium was found to have strong anti-tumor activity on four cancer cell lines, MCF-7 breast cancer, B16 mouse melanoma, HL-60 human premyelocytic leukemia and HepG2 human hepatocellular carcinoma [19]. Some specific fractions of extracts of $C$. sinensis, especially polysaccharides have been found to modify immune response more precisely. The conditioned medium from the polysaccharide fraction of $C$. sinensis (PSCS)-stimulated blood mononuclear cells [PSCS-MNC-CM] had an activity that could significantly inhibit the proliferation of human leukemic U937 cells resulting in a growth inhibition rate of 78$83 \%$. Furthermore, PSCS-MNC-CM treatment induced about $50 \%$ of the cells differentiating into mature monocytes/macrophages expressing nonspecific esterase (NSE) and the surface antigens of CD11b, CD14 and CD 68. The levels of interferon (IFN)-gamma, tumor necrosis factor (TNF)-alpha and interleukin (IL)-1 were very low in normal MNC-CM but they were greatly increased in MNC-CM prepared with PSCS stimulation. Antibody neutralization studies further revealed that the tumoricidal and differentiating effects of PSCS-MNC-CM were mainly derived from the elevated cytokines, especially IFN-gamma and TNF-alpha [20]. An exopolysaccharide fraction (EPSF), prepared from C. sinensis significantly enhanced the phagocytosis capacity of peritoneal macrophages and proliferation ability of spleen lymphocytes of $\mathrm{H} 22$ tumor (histocompatibility 22) and B16 melanoma bearing mice, as well as inhibited the tumor growth in separate study. EPSF significantly promoted macrophages' TNF-alpha expression and spleen lymphocytes' cytotoxicity. EPSF also significantly elevated TNFalpha and IFN-gamma mRNA expression of splenic lymphocytes and thus elevated immunocytes' activity in H22 tumor bearing mice $[21,22]$. The EPSF from $C$. sinensis also has inhibitory effect on oncogene expression. The c-Myc, cFos, and vascular endothelial growth factor (VEGF) levels in the lungs and livers of EPSF-treated mice were found to be significantly lower than those of untreated mice [23]. When lipopolysaccharide (LPS)-activated murine macrophage cell line R309 was exposed to the extracts $C$. sinensis, R309 induced significant levels of IL-1. IL-2 induction was recognized in T cell line LBRM-33 1A5 (1A5) cultures in the presence of IL-1 and phytohemagglutinin (PHA). However, no enhancement of IL-2 production by $C$. sinensis was discerned in $1 \mathrm{~A} 5$ cultures with IL-1 and PHA, i.e., direct action of $C$. sinensis was not found on IL-2 production of $1 \mathrm{~A} 5$. PHA-stimulated 1A5 exposed to C. sinensis induced IL-2 without IL-1 when co-cultured with LPS-activated R309 as a source of IL-1 [24]. C. sinensis, either alone or with IFNgamma induction, increased the MHC class II antigen expression on hepatoma cell line HA22T/VGH, which makes the host immune surveillance more effective against tumor cells with down-regulated MHC class II antigen expression [25]. In leukemia patients, $C$. sinensis augmented the NK cell activity and also improved the CD16 marker expression on lymphocytes and the binding capacity to K562 cells [26]. In breast cancer, the oral $C$. sinensis did not reduce primary tumor growth but reduced lung metastasis occurrence in a surgical excision model of metastatic mammary carcinoma.
The reduction in metastases growth is supposed to due to the effects of macrophage-derived factors on tumor cell cycle, NK cell activation and other immunostimulating activities $[27,28]$.

Although the immunomodulating activity of $C$. sinensis is mainly responsible for its anticancer activity, it is not so simply described and only exact mechanism. Directly or indirectly many other metabolic and genetic pathways are responsible. Extensive research works have been done to evaluate the mechanism of anticancer activity of $C$. sinensis and the most significant mechanism has been found to influence apoptosis. In a study, the ethyl acetate extract of mycelium of $C$. sinensis induced the characteristic apoptotic symptoms in human premyelocytic leukemia cells (HL-60), DNA fragmentation and chromatin condensation. The activation of caspase- 3 and the specific proteolytic cleavage of poly ADP-ribose polymerase were detected during the course of apoptosis induction. These results suggest that this extract inhibited cancer cell proliferation by inducing cell apoptosis [29]. The involvement of caspase- 8 with caspase-3 was also found in $C$. sinensis induced apoptosis in MA-10 mouse Leydig tumor cells [30]. Components from Cordyceps induce tumor cell apoptosis through both extrinsic and intrinsic pathways. Two new epipolythiodioxopiperazines, named gliocladicillins A and B, from Cordyceps-colonizing fungi inhibited growth of HeLa, HepG2 and MCF-7 tumor cells by arresting the cell cycle at $\mathrm{G}(2) / \mathrm{M}$ phase and induced apoptosis through up-regulation of expression of p53, p21, and cyclin $\mathrm{B}$ and activation of caspases-8, -9 and -3 [31]. Furthermore, EPSF of $C$. sinensis decreased the levels of Bcl-2 in the lungs and livers [22]. The antitumor activity of C. sinensis by inducing apoptosis was also found in human colorectal (HT-29 and HCT 116), hepatocellular (Hep 3B and Hep G2) carcinoma cells [32] and human oral squamous cancer cell line (OEC-M1) [33]. Interestingly, Tang et al. [34] reported that $C$. sinensis reduced Angiotensin II induced NRK-52E cell apoptosis, which may be part of its mechanism of the protective effects on hypertensive renal damage.

The antioxidant activity of $C$. sinensis components is also responsible for its anticancer property. The ethanolic extract of $C$. sinensis was found to have inhibitory effect on lipid peroxidation and protective effect on 4-nitroquinoline oxidetriggered DNA lesion in V79 hamster cells [35]. The polysaccharide component of $C$. sinensis has been found to inhibit the tumor growth of H22-bearing mice by modulating the antioxidant enzymes activity such as enhancing superoxide dismutase (SOD) activity of liver, brain and serum as well as glutathione peroxidase (GPx) activity of liver and brain in tumor-bearing mice [36]. Wang et al. [32] reported the free radical scavenging activity of $C$. sinensis. As free radicals are responsible for oxidative damage of apoptotic genes, by scavenging free radicals, $C$. sinensis protects apoptotic genes and induces the apoptosis of cancer cells.

\section{ANTICANCER ACTIVITY OF CORDYCEPS MILITARIS}

The mushroom C. militaris has been used for a long time in eastern Asia as a nutraceutical and in traditional Chinese medicine as a therapeutic agent for cancer patients. C. militaris has been found good for inhibiting the growth of tumor, prolonging the survival period of mice implanted with S180 
sarcoma cells and inhibiting the growth and metastasis of Lewis pneumonic cancer in the implanted mice [37]. Water extract of $C$. militaris inhibited growth of human umbilical vein endothelial cells (HUVEC) and human sarcoma cell line HT1080. This mushroom extract also reduced metalloproteinase 2 (mmp2) gene expression in HT1080 and basic fibroblast growth factor (bFGF) gene expression in HUVECs [38]. Recently, a cytotoxic protease was purified from the dried fruiting bodies of $C$. militaris, which exhibited cytotoxicity against human breast and bladder cancer cells [39].

Like $C$. sinensis, the most significant anticancer mechanism of $C$. militaris was found by inducing cell apoptosis. In a study, Park et al. [40] observed that the aqueous extract of C. militaris (AECM) inhibited cell growth of human leukemia U937 cells by morphological change and apoptotic cell death such as formation of apoptotic bodies and DNA fragmentation. They also observed the down-regulation of antiapoptotic gene bcl-2 expression and proteolytic activation of caspase-3 in AECM-treated U937 cells. But AECM did not affect the pro-apoptotic gene bax expression and activity of caspase-9. The hot water extract of $C$. militaris was also found to induce apoptosis in the human premyelocytic leukemia HL-60 cells and the activation of caspase- 3 and the specific proteolytic cleavage of Poly ADP-ribose polymerase (PARP) were detected during the course of apoptosis [41]. In addition to the activation of caspase-3, the AECM-induced apoptosis may relate to the inactivation of Akt (an oncogene) in human breast cancer MDA-MB-231 cells [42]. In another study, the growth inhibition and apoptosis induction by the water extract of $C$. militaris (WECM) treatment in human lung carcinoma A549 cells were found associated with the induction of Fas expression, catalytic activation of caspase8, and Bid cleavage. Activation of caspases, downregulation of anti-apoptotic gene bcl-2 expression, and upregulation of pro-apoptotic Bax protein were also observed in WECMtreated cancer cells. In addition, WECM exerted a dosedependent inhibition of telomerase activity via down-regulation of human telomerase reverse transcriptase (hTERT), c-Myc and Sp1 expression. The data indicated that WECM induced the apoptosis of A549 cells through a signaling cascade of death receptor-mediated extrinsic and mitochondriamediated intrinsic caspase pathways and diminishing the telomerase activity through the inhibition of hTERT transcriptional activity [43].

\section{ANTICANCER ACTIVITY OF OTHER CORDYCEPS SPECIES}

Three different polysaccharide-peptide complexes (PPCs) were produced by submerged mycelial culture of a rare entomopathogenic fungus Cordyceps sphecocephala and their anticancer activities were investigated in human hepatocarcinoma (HepG2) and neuroblastoma (SK-N-SH) cells. In this study, PPC-induced apoptosis of both cancer cells was found which was associated with intracellular events including DNA fragmentation, activation of caspase3 , and modulation of Bcl-2 and Bax and no cytotoxicity against normal cells was reported [44].

A water-insoluble extracellular glucan (CO-1) was isolated from the precipitate formed on incubation of the culture filtrate of Cordyceps ophioglossoides and this CO-1 strongly inhibited the growth of Sarcoma 180 solid-type tumor [45]. The effects of protein-bound polysaccharide (SN-C) extracted from $C$. ophioglossoides on the growth of transplanted allogeneic and syngeneic murine tumors were studied and it was found that SN-C given by intraperitoneal administration suppressed the growth of sarcoma-180 transplanted subcutaneously in mice. SN-C also showed a significant cytocidal effect on cultured tumor cells but did not affect delayed-type hypersensitivity (DTH) in normal mice [46]. A protein-bound galactosaminoglycan (CO-N) was isolated from $\mathrm{SN}-\mathrm{C}$ of $C$. ophioglossoides. When given intraperitoneally to mice, $\mathrm{CO}-\mathrm{N}$ inhibited the proliferation of sarcoma 180 cells inoculated into the peritoneal cavity and exhibited a marked life-prolonging effect against ascitic tumors such as Ehrlich carcinoma. CO-N also showed an inhibitory effect against solid Ehrlich carcinoma when given intratumorally and significantly inhibited the growth of a syngeneic solid tumor (MM46 mammary carcinoma) upon intravenous administration at a low dose [47].

\section{CORDYCEPIN: AN ANTICANCER AGENT FROM CORDYCEPS SPECIES}

Cordycepin, or 3'-deoxyadenosine, is a derivative of the nucleoside adenosine, differing from adenosine by the absence of oxygen in the 3' position of its ribose part. Cordycepin was isolated from the water extract of $C$. sinensis $[33,48]$. Later the major component of the butanol fraction of $C$. militaris was also identified as cordycepin by high performance liquid chromatography [49]. Because cordycepin is similar to adenosine, RNA polymerase cannot discriminate between the two and when incorporated into a growing RNA molecule, cordycepin prevents further elongation, thus producing a prematurely terminated RNA molecule [50].

Orally administered cordycepin inhibited B16-BL6 melanoma cell growth in mice with no adverse effects [48]. Further study demonstrated that cordycepin inhibited the proliferation of B16-BL6 cells by stimulating adenosine A3 receptors followed by the Wnt signaling pathway, including GSK-3beta activation and cyclin D1 inhibition [51]. Cordycepin markedly inhibited the phosphorylation of Akt and p38 in dose-dependent manners in LPS-activated macrophage. Moreover, cordycepin suppressed TNF-alpha expression, Ikappa B alpha phosphorylation and translocation of nuclear factor-kappa B (NF-kappa B) [49]. Cordycepin-induced apoptosis is also reported. In MA-10 cells (a mouse Leydig tumor cell line), cordycepin induced DNA fragmentation, declined the percentage of G1 and G2/M phase cells, increased the percentages of subG1 phase cells suggesting cordycepin induced MA-10 cell apoptosis. Moreover, western blotting analysis showed that cordycepin induced caspase-9, caspase--3 and caspase--7 protein expressions, but not caspase-8 [52]. In another study, cordycepin significantly induced cell apoptosis in OEC-M1 human oral squamous cancer cells [23]. It was also suggested that the effect of cordycepin on the growth of tumor cells was significantly related to the metabolism-associated protein expression induced by cordycepin [53]. Platelet aggregation induced by cancer cells is an indispensable step for hematogenic metastasis and it was showed that cordycepin had an inhibitory effect on hematogenic metastasis of B16-F1 melanoma cells 
via blocking of ADP-induced platelet aggregation in vivo [54]. A novel molecular mechanism for the anti-tumor effects of cordycepin in two different bladder cancer cell lines, 5637 and T-24 cells has been revealed by Lee et al. [55]. They reported that cordycepin treatment during cell-cycle progression resulted in significant growth inhibition, which was largely due to G2/M-phase arrest and resulted in an upregulation of p21WAF1 expression, independent of the p53 signaling pathway. Moreover, treatment with cordycepin induced phosphorylation of JNK (c-Jun N-terminal kinases). Blockade of JNK function using SP6001259 (JNK-specific inhibitor) and small interfering RNA (si-JNK1) rescued cordycepin-dependent p21WAF1 expression, inhibited cell growth and decreased cell cycling proteins.

Derivatives of cordycepin have also showed anticancer activity. In an experiment, 1-O-Acetyl-2,5-di-O-p-chlorobenzoyl-3-deoxy-D-ribofuranose, derived from cordycepin was coupled with trimethylsilylated derivatives of N4propionylcytosine, N4-p-toluoyl-5-fluorocytosine and 5fluorouracil in the presence of trimethylsilyl trifluoromethanesulfonate (TMS-triflate) to give fully acylated nucleosides. Selective removal of the N4-propionyl group of these nucleosides by treatment with hydrazine hydrate gave 2',5'-di-O-p-chlorobenzoyl-3'-deoxycytidine, deamination of which with sodium nitrite in trifluoroacetic acid afforded 2',5'-di-O-p-chlorobenzoyluridine in good yield. The acylated nucleosides and 2',5'-di-O-p-chlorobenzoyluridine were saponified to give free 3 '-deoxycytidine, 5-fluoro-3'-deoxycytidine, 3'-deoxyuridine, and 5-fluoro-3'-deoxyuridine, respectively. These 3'-deoxyribonucleosides were then examined for growth-inhibitory effects on mouse leukemic L5178Y cells, and their IC50 (inhibitory concentration 50) values were found $1.8,33,6.5$, and 18 (microgram $/ \mathrm{ml})$ respectively [56]. Other nucleotide derivatives also exist which showed anticancer activities, such as, forodesine (also known as Immucillin $\mathrm{H}$; IUPAC name: 7-[(2S,3S,4R,5R)3,4-dihydroxy-5-(hydroxymethyl)-2-pyrrolidinyl]-1,5-dihydropyrrolo[2,3-e]pyrimidin-4-one) is a inhibitor of purine nucleoside phosphorylase, which is recognized as a potential target for the treatment of patients with T-cell malignancies and also B-cell chronic lymphocytic leukemia $[57,58]$.

\section{CONCLUSIONS}

Currently, in some parts of the world, there is a renaissance of interest in traditional remedies. Most of the investigators believe that traditional medicine is a promising source of new therapeutics. Mushrooms have a notable place in folklore or traditional medicine throughout the world. Medicinal mushrooms are now the subject of study for many ethnobotanists and medical researchers. The ability of some mushrooms, especially Cordyceps to inhibit tumor growth and enhance aspects of the immune system has been a subject of research for approximately 50 years. As cancer is one of the most complicated diseases and its pathogenic mechanism is diverse, therapeutic attempts are being taken against cancer from different angles. The cancer protecting activities of Cordyceps are widely reported and established and now it is the time to look for the cancer preventing activity of this mushroom more precisely. Extensive research with Cordyceps mushrooms may contribute to the discovery of new anticancer strategies.

\section{ACKNOWLEDGEMENTS}

The work was supported by the grant No.30371660 from the National Natural Science Foundation of China and the grant CMB No.99-698 from China Medical Board of New York, INC.

\section{REFERENCES}

[1] Wasser SP, Weis AL. Medicinal properties of substances occurring in higher Basidiomycetes mushrooms: Current perspectives. Int $\mathbf{J}$ Med Mushr 1999; 1(1): 31-62.

[2] Kaul TN. Biology and Conservation of mushrooms. New Delhi: Oxford \& IBH Publishing Co. Pvt. Ltd 2001: pp. 117-45.

[3] Chihara G, Hamuro J, Maeda Y, Arai Y, Fukuoka F. Fractionation and purification of the polysaccharides with marked antitumor activity, especially lentinan, from Lentinus edodes (Berk.) Sing. (an edible mushroom). Cancer Res 1970; 30(11): 2776-81.

[4] Kabir Y, Yamaguchi M, Kimura S. Effect of shiitake (Lentinus edodes) and maitake (Grifola frondosa) mushrooms on blood pressure and plasma lipids of spontaneously hypertensive rats. J Nutr Sci Vitaminol (Tokyo) 1987; 33(5): 341-6.

[5] Hirasawa M. Three kinds of antibacterial substances from Lentinan edodes (Berk) Sing. (Shiitake, an edible mushroom). Int J Antimicrob Agents 1999; 11(2): 151-7.

[6] Kim H, Kacew S, Lee B. In vitro chemopreventive effects of plant polysaccharides (Aloe barbadensis miller, Lentinus edodes, Ganoderma lucidum and Coriolus versicolor). Carcinogenesis 1999; 20(8): 1637-40.

[7] Yoon SY, Eo SK, Kim YS, Lee CK, Han SS. Antimicrobial activity of Ganoderma lucidum extract alone and in combination with some antibiotics. Arch Pharm Res 1994; 17(6): 438-42.

[8] el-Mekkawy S, Meselhy MR, Nakamura N, et al. Anti-HIV-1 and anti-HIV-1-protease substances from Ganoderma lucidum. Phytochemistry 1998; 49(6): 1651-7.

[9] Shieh YH, Liu CF, Huang YK, et al. Evaluation of the hepatic and renal-protective effects of Ganoderma lucidum in mice. Am J Clin Med 2001; 29(3): 501-7.

[10] Hossain S, Hashimoto M, Choudhury EK, et al. Dietary mushroom (Pleurotus ostreatus) ameliorates atherogenic lipid in hypercholesterolaemic rats. Clin Exp Pharmacol Physiol 2003; 30(7): 470-5.

[11] Alam N, Amin R, Khan A, et al. Comparative effects of oyster mushrooms on lipid profile, liver and kidney function in hypercholesterolemic rats. Mycobiology 2009; 37(1): 37-42.

[12] Holliday J, Cleaver M. Medicinal value of the caterpillar fungi species of the genus cordyceps (Fr.) Link (Ascomycetes): a Review. Int J Med Mushr 2008; 10(3): 219-34.

[13] Mizuno T. Medicinal effects and utilization of Cordyceps (Fr.) Link (Ascomycetes) and Isaria Fr. (Mitosporic fungi) Chinese caterpillar fungi, "Tochukaso". Int J Med Mushr 1999; 1(1): 251-62.

[14] Coates PM, Blackman MR, Cragg G, Levine M, Moss J, White J, Eds. Encyclopedia of dietary supplements. New York: Marcel Dekker 2005; pp. 1-13.

[15] Yoshida J, Takamura S, Yamaguchi N, et al. Antitumor activity of an extract of Cordyceps sinensis (Berk.) Sacc. against murine tumor cell lines. Jpn J Exp Med 1989; 59(4): 157-61.

[16] Nakamura K, Konoha K, Yamaguchi Y, Kagota S, Shinozuka K, Kunitomo M. Combined effects of Cordyceps sinensis and methotrexate on hematogenic lung metastasis in mice. Receptors Channels 2003; 9(5): 329-34.

[17] Xu RH, Peng XE, Chen GZ, Chen GL. Effects of cordyceps sinensis on natural killer activity and colony formation of B16 melanoma. Chin Med J (Engl) 1992; 105(2): 97-101.

[18] Shin KH, Lim SS, Lee S, Lee YS, Jung SH, Cho SY. Anti-tumour and immuno-stimulating activities of the fruiting bodies of Paecilomyces japonica, a new type of Cordyceps spp. Phytother Res 2003; 17(7): 830-3.

[19] Wu JY, Zhang QX, Leung PH. Inhibitory effects of ethyl acetate extract of Cordyceps sinensis mycelium on various cancer cells in culture and B16 melanoma in C57BL/6 mice. Phytomedicine 2007; 14(1): 43-9.

[20] Chen YJ, Shiao MS, Lee SS, Wang SY. Effect of Cordyceps sinensis on the proliferation and differentiation of human leukemic U937 cells. Life Sci 1997; 60(25): 2349-59.

[21] Zhang W, Yang J, Chen J, Hou Y, Han X. Immunomodulatory and antitumour effects of an exopolysaccharide fraction from cultivated 
Cordyceps sinensis (Chinese caterpillar fungus) on tumour-bearing mice. Biotechnol Appl Biochem 2005; 42(1): 9-15.

[22] Zhang W, Li J, Qiu S, Chen J, Zheng Y. Effects of the exopolysaccharide fraction (EPSF) from a cultivated Cordyceps sinensis on immunocytes of H22 tumor bearing mice. Fitoterapia 2008; 79(3): 168-73.

[23] Yang J, Zhang W, Shi P, Chen J, Han X, Wang Y. Effects of exopolysaccharide fraction (EPSF) from a cultivated Cordyceps sinensis fungus on c-Myc, c-Fos, and VEGF expression in B16 melanoma-bearing mice. Pathol Res Pract 2005; 201(11): 745-50.

[24] Kawanishi T, Ikeda-Dantsuji Y, Nagayama A. Effects of two basidiomycete species on interleukin 1 and interleukin 2 production by macrophage and T cell lines. Immunobiology 2009 November; [Epub ahead of print; doi:10.1016/j.imbio.2009.10.005].

[25] Chiu $\mathrm{JH}, \mathrm{Ju} \mathrm{CH}, \mathrm{Wu} \mathrm{LH}$, et al. Cordyceps sinensis increases the expression of major histocompatibility complex class II antigens on human hepatoma cell line HA22T/VGH cells. Am J Chin Med 1998; 26(2): 159-70.

[26] Liu C, Lu S, Ji MR. Effects of Cordyceps sinensis (CS) on in vitro natural killer cells. Zhongguo Zhong Xi Yi Jie He Za Zhi 1992; 12(5): 267-9.

[27] Yoon TJ, Yu KW, Shin KS, Suh HJ. Innate immune stimulation of exo-polymers prepared from Cordyceps sinensis by submerged culture. Appl Microbiol Biotechnol 2008; 80(6): 1087-93.

[28] Jordan JL, Nowak A, Lee TD. Activation of innate immunity to reduce lung metastases in breast cancer. Cancer Immunol Immunother 2009; 59(5): 789-97.

[29] Zhang Q, Wu J, Hu Z, Li D. Induction of HL-60 apoptosis by ethyl acetate extract of Cordyceps sinensis fungal mycelium. Life Sci 2004; 75(24): 2911-9.

[30] Yang HY, Leu SF, Wang YK, Wu CS, Huang BM. Cordyceps sinensis mycelium induces MA-10 mouse Leydig tumor cell apoptosis by activating the caspase- 8 pathway and suppressing the NF-kappaB pathway. Arch Androl 2006; 52(2): 103-10.

[31] Chen Y, Guo H, Du Z, Liu XZ, Che Y, Ye X. Ecology-based screen identifies new metabolites from a Cordyceps-colonizing fungus as cancer cell proliferation inhibitors and apoptosis inducers. Cell Prolif 2009; 42(6): 838-47.

[32] Wang BJ, Won SJ, Yu ZR, Su CL. Free radical scavenging and apoptotic effects of Cordyceps sinensis fractionated by supercritical carbon dioxide. Food Chem Toxicol 2005; 43(4): 543-52.

[33] Wu WC, Hsiao JR, Lian YY, Lin CY, Huang BM. The apoptotic effect of cordycepin on human OEC-M1 oral cancer cell line. Cancer Chemother Pharmacol 2007; 60(1): 103-11.

[34] Tang R, Zhou Q, Shu J, et al. Effect of Cordyceps sinensis extraxt on Klotho expression and apoptosis in renal tubular epithelial cells induced by angiotensin II. J Cent South Univ (Med Sci) 2009; 34(4): 300-7.

[35] Ra Yoon M, Hyun Nam S, Young Kang M. Antioxidative and antimutagenic activities of $70 \%$ ethanolic extracts from four fungal mycelia-fermented specialty rices. J Clin Biochem Nutr 2008; 43(2): 118-25.

[36] Chen J, Zhang W, Lu T, Li J, Zheng Y, Kong L. Morphological and genetic characterization of a cultivated Cordyceps sinensis fungus and its polysaccharide component possessing antioxidant property in H22 tumor-bearing mice. Life Sci 2006; 78(23): 27428.

[37] Liu J, Yang S, Yang X, Chen Z, Li J. Anticarcinogenic effect and hormonal effect of Cordyceps militaris Link. Zhongguo Zhong Yao Za Zhi 1997; 22(2): 111-3.

[38] Yoo HS, Shin JW, Cho JH, et al. Effects of Cordyceps militaris extract on angiogenesis and tumor growth. Acta Pharmacol Sin 2004; 25(5): 657-65.

[39] Park BT, Na KH, Jung EC, Park JW, Kim HH. Antifungal and anticancer activities of a protein from the mushroom Cordyceps militaris. Korean J Physiol Pharmacol 2009; 13(1): 49-54.

[40] Park C, Hong SH, Lee JY, et al. Growth inhibition of U937 leukemia cells by aqueous extract of Cordyceps militaris through induction of apoptosis. Oncol Rep 2005; 13(6): 1211-6.
[41] Lee H, Kim YJ, Kim HW, Lee DH, Sung MK, Park T. Induction of apoptosis by Cordyceps militaris through activation of caspase-3 in leukemia HL-60 cells. Biol Pharm Bull 2006; 29(4): 670-4.

[42] Jin CY, Kim GY, Choi YH. Induction of apoptosis by aqueous extract of Cordyceps militaris through activation of caspases and inactivation of Akt in human breast cancer MDA-MB-231 Cells. J Microbiol Biotechnol 2008; 18(12): 1997-2003.

[43] Park SE, Yoo HS, Jin CY, et al. Induction of apoptosis and inhibition of telomerase activity in human lung carcinoma cells by the water extract of Cordyceps militaris. Food Chem Toxicol 2009; 47(7): 1667-75.

[44] Oh JY, Baek YM, Kim SW, et al. Apoptosis of human hepatocarcinoma (HepG2) and neuroblastoma (SKN-SH) cells induced by polysaccharides-peptide complexes produced by submerged mycelial culture of an entomopathogenic fungus Cordyceps sphecocephala. J Microbiol Biotechnol 2008; 18(3): 512-9.

[45] Yamada H, Kawaguchi N, Ohmori T, Takeshita Y, Taneya S, Miyazaki T. Structure and antitumor activity of an alkali-soluble polysaccharide from Cordyceps ophioglossoides. Carbohydr Res 1984; 125(1): 107-15.

[46] Ohmori T, Tamura K, Tsuru S, Nomoto K. Antitumor activity of protein-bound polysaccharide from Cordyceps ophioglossoides in mice. Jpn J Cancer Res 1986; 77(12): 1256-63.

[47] Ohmori T, Tamura K, Fukui K, et al. Isolation of galactosaminoglycan moiety $(\mathrm{CO}-\mathrm{N})$ from protein-bound polysaccharide of Cordyceps ophioglossoides and its effects against murine tumors. Chem Pharm Bull 1989; 37(4): 1019-22.

[48] Yoshikawa N, Nakamura K, Yamaguchi Y, Kagota S, Shinozuka K, Kunitomo M. Antitumour activity of cordycepin in mice. Clin Exp Pharmacol Physiol 2004; 31(2): 51-3.

[49] Kim HG, Shrestha B, Lim SY, et al. Cordycepin inhibits lipopolysaccharide-induced inflammation by the suppression of NF-kappaB through Akt and p38 inhibition in RAW 264.7 macrophage cells. Eur J Pharmacol 2006; 545(2):192-9.

[50] Siev M, Weinberg R, Penman S. The selective interruption of nucleolar RNA synthesis in HELA cells by cordycepin. J Cell Biol 1969; 41(2): 510- 20.

[51] Yoshikawa N, Yamada S, Takeuchi C, et al. Cordycepin (3'deoxyadenosine) inhibits the growth of B16-BL6 mouse melanoma cells through the stimulation of adenosine A3 receptor followed by glycogen synthase kinase-3beta activation and cyclin D1 suppression. Naunyn Schmiedebergs Arch Pharmacol 2008; 377(4): 591-5.

[52] Jen CY, Lin CY, Leu SF, Huang BM. Cordycepin Induced MA-10 Mouse Leydig Tumor Cell Apoptosis through Caspase-9 Pathway. Evid Based Complement Alternat Med 2009 January; [Epub ahead of print; doi: 10.1093/ecam/nen084].

[53] Shi P, Huang Z, Tan X, Chen G. Proteomic detection of changes in protein expression induced by cordycepin in human hepatocellular carcinoma BEL-7402 cells. Methods Find Exp Clin Pharmacol 2008; 30(5): 347-53.

[54] Yoshikawa N, Kunitomo M, Kagota S, Shinozuka K, Nakamura K. Inhibitory effect of cordycepin on hematogenic metastasis of B16F1 mouse melanoma cells accelerated by adenosine-5'-diphosphate. Anticancer Res 2009; 29(10): 3857-60.

[55] Lee SJ, Kim SK, Choi WS, Kim WJ, Moon SK. Cordycepin causes p21WAF1-mediated G2/M cell-cycle arrest by regulating c-Jun Nterminal kinase activation in human bladder cancer cells. Arch Biochem Biophys 2009; 490(2):103-9.

[56] Saneyoshi M, Kohsaka-Ichikawa M, Yahata A, Kimura S, Izuta S, Yamaguchi T. Synthetic nucleosides and nucleotides. XXXV. Synthesis and biological evaluations of 5-fluoropyrimidine nucleosides and nucleotides of 3-deoxy-beta-D-ribofuranose and related compounds. Chem Pharm Bull (Tokyo) 1995; 43(11): 2005-9.

[57] Gandhi V, Balakrishnan K. Pharmacology and mechanism of action of forodesine, a T-cell targeted agent. Semin Oncol 2007; 34: S8-S12.

[58] Kicska GA, Long L, Hörig H, et al. Immucillin H, a powerful transition-state analog inhibitor of purine nucleoside phosphorylase, selectively inhibits human T lymphocytes. Proc Natl Acad Sci USA 2001; 98(8): 4593-8. 